\title{
Clinicoradiological manifestations of paraganglioma syndromes associated with succinyl dehydrogenase enzyme mutation
}

\author{
Brendan Barber • Mark Ingram • Sameer Khan • \\ Gul Bano • Shirley Hodgson • Ioannis Vlahos
}

Received: 15 November 2010 /Revised: 16 March 2011 /Accepted: 4 April 2011 /Published online: 22 April 2011

(C) European Society of Radiology 2011

\begin{abstract}
Background Paragangliomas are rare tumours derived from the autonomic nervous system that have increasingly been recognised to have a genetic predisposition. Mutations of the enzyme succinyl dehydrogenase (SDH) have proven to result in paraganglioma formation. There are four subunits (A through D) that form the enzyme complex and are associated with different genophenotypic expressions of disease. SDHB and SDHD mutations are more common, whereas SDHA and SDHC mutations are rare. Patients with SDHB mutations are prone to extra-adrenal pheochromocytomas, malignant disease and extra-paraganglial neoplasia, whereas SDHD mutations have a greater propensity for multiple, benign head and neck paragangliomas.
\end{abstract}

\footnotetext{
B. Barber $(\bowtie) \cdot$ M. Ingram $\cdot$ I. Vlahos

Radiology Department, St George's Hospital,

Blackshaw Road,

London SW17 0QT, UK

e-mail: Brendan.Barber@stgeorges.nhs.uk

S. Khan

Radiology Department, Charing Cross Hospital,

Fulham Palace Road,

London W6 8RF, UK

G. Bano

Endocrine Department, St George's Hospital,

Blackshaw Road,

London SW17 0QT, UK

S. Hodgson

Genetics Department, St George's Hospital,

Blackshaw Road,

London SW17 0QT, UK
}

Methods Diagnosis of a sporadic paraganglioma or pheochromocytoma should lead to a full genetic workup of the patient and family if SDH mutations are found.

Results Further annual screening will be required depending on the mutation, which can have a significant impact on radiologists and the resources of the radiology department. Conclusion We present our imaging experience with a series of patients with proven SDH mutations resulting in paragangliomas with a review of the literature.

Keywords Paragangliomas · Imaging · Succinyl · Dehydrogenase $\cdot$ Mutations

\section{Introduction}

Paragangliomas are rare tumours derived from the autonomic nervous system. Traditionally these tumours have been considered to be either "sporadic" or "hereditary". Increasingly many adrenal paragangliomas have been recognised to have a genetic predisposition. One recently recognised genetic predisposition relates to mutations of the enzyme succinyl dehydrogenase (SDH). SDH is an enzyme involved in the Krebs cycle and the production of intracellular energy. Recently discovered mutations of the SDH enzyme have proven to result directly in paraganglioma formation, and tumours previously thought to be sporadic may, therefore, be hereditary. Additionally these paragangliomas may be associated with neoplasms in other organs. These developments in genetics have implications for radiology with regard to screening of patients and their families for tumours. We present our imaging experience with a series of patients with proven SDH mutations resulting in paragangliomas with a review of the literature. 


\section{Content}

Embryology

Paraganglia are aggregations of cells derived from the neural crest. They are located throughout the body in vascular and neuronal adventitia from the skull base to pelvic floor [1], with the adrenal medulla being the largest
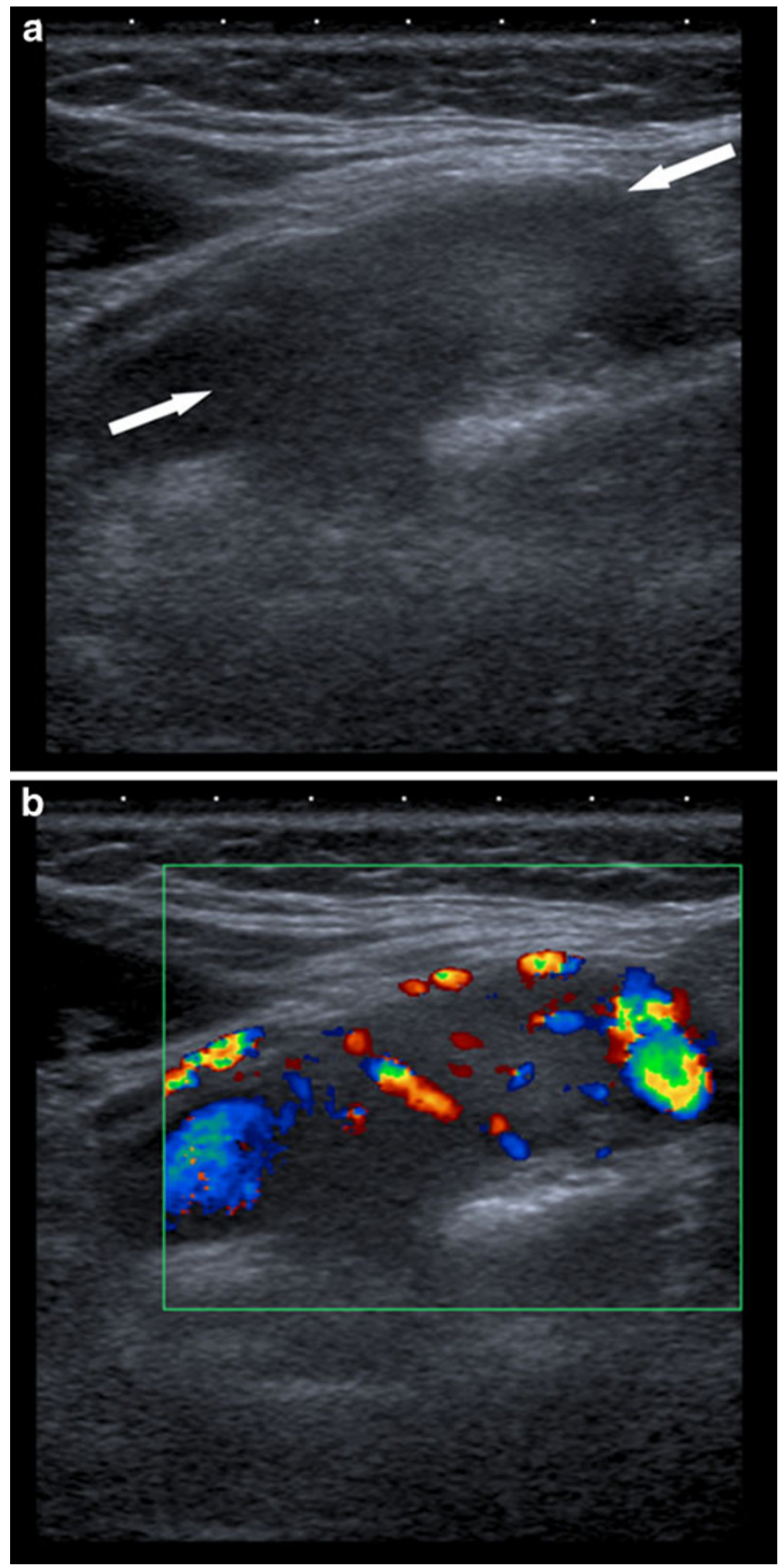

Fig. 1 A 56-year-old female patient with a right-sided carotid paraganglioma of the neck. Doppler ultrasound images. The paraganglioma appears as a homogeneous hypoechoic mass (between the arrows). Doppler imaging reveals multidirectional hypervascularity, which is a discerning feature of paragangliomas focal collection. Paraganglia are generally associated with either the parasympathetic or sympathetic nervous system. Although the functions of the parasympathetic and sympathetic paraganglia overlap, the parasympathetic paraganglia predominantly have a chemoreceptor function, whilst the sympathetic paraganglia are predominantly secretory in function. The parasympathetic paraganglia are located in the head, neck and anterior mediastinum, whereas the sympathetic paraganglia are located in the posterior mediastinum and paravertebral retroperitoneum. Further paraganglia are found around viscera such as the bladder [2]. This functional division relates to the various SDH subunit mutation clinical presentations.

\section{Terminology}

The term paragangliomas encompasses all neoplasms arising from the parasympathetic and sympathetic auto-
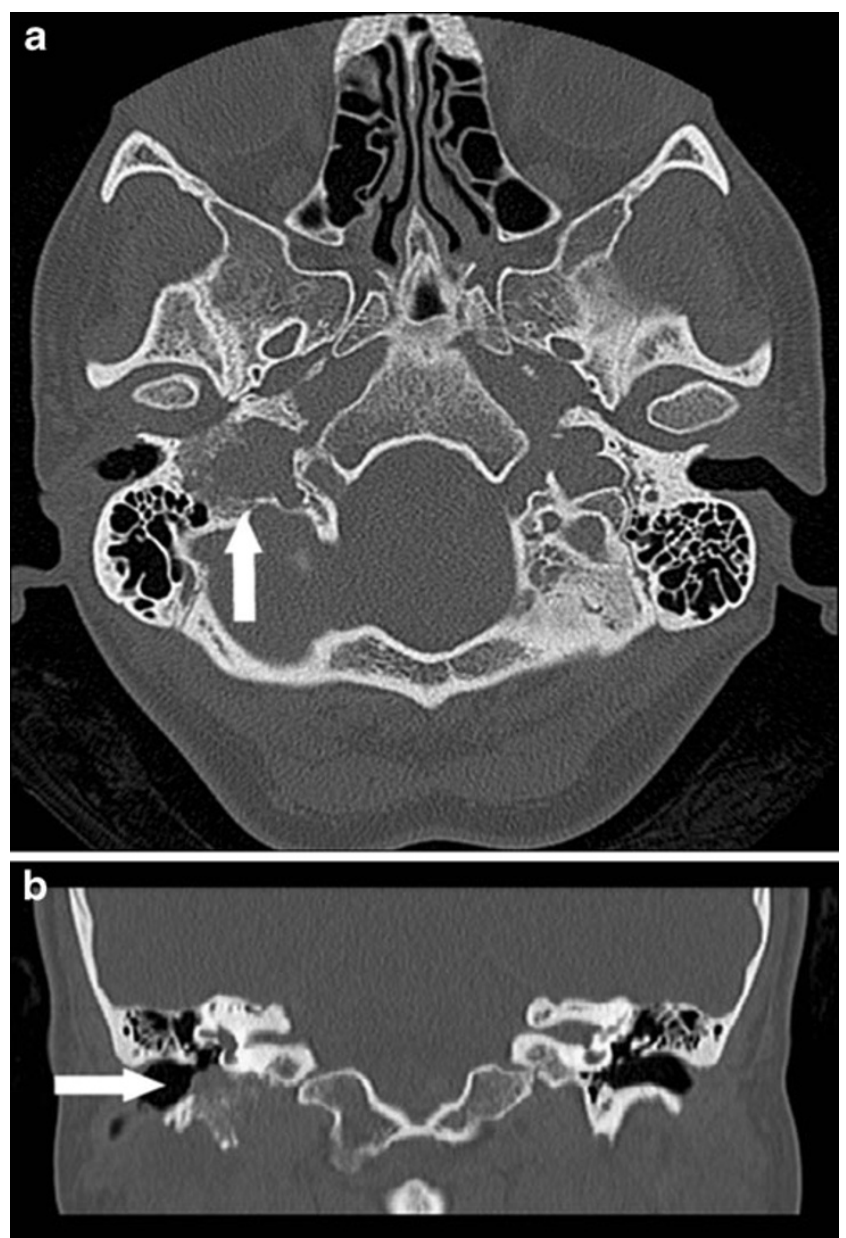

Fig. 2 A 35-year-old female patient with SDHD and a right jugulotympanic paraganglioma. a Axial CT with bone windowing demonstrates a soft tissue lesion within the jugulotympanic fossa causing surrounding bone erosion (arrow). b Coronal CT reformat with bone windowing demonstrating the soft tissue mass extending into the external auditory canal and bone erosion (arrow) 
nomic system, irrespective of their site of origin. Paragangliomas associated with the sympathetic system are also generally secretory in nature, and historically, those arising in the adrenal medulla have been called pheochromocytomas, whereas those arising outside the adrenal have been termed extra-adrenal pheochromocytomas [3]. More recently, the 2004 World Health Organisation classification of endocrine tumours defined a pheochromocytoma as an intra-adrenal paraganglioma and classified extra-adrenal sympathetic tumours as extraadrenal paragangliomas [4].

Parasympathetic paragangliomas of the head and neck also have a number of historical names such as chemodectoma and glomus tumours, but are currently referred to by their location, e.g., carotid paraganglioma. Perhaps confusingly, similar to the secretory sympathetic tumours these extra-adrenal non-secretory parasympathetic lesions are also collectively defined by the 2004 WHO classification as extra-adrenal paragangliomas [4].

\section{Role of SDH and genetic predisposition}

Succinate dehydrogenase (SDH) is an enzyme complex involved in the tricarboxylic acid cycle. It is also known as mitochondrial complex II and forms part of the electron transport train. There are four subunits (A through to D), which form the enzyme complex. The exact pathogenetic mechanism whereby the subunit mutations result in paraganglioma formation is not known [5]. It has long been recognised that there is an increased incidence of carotid

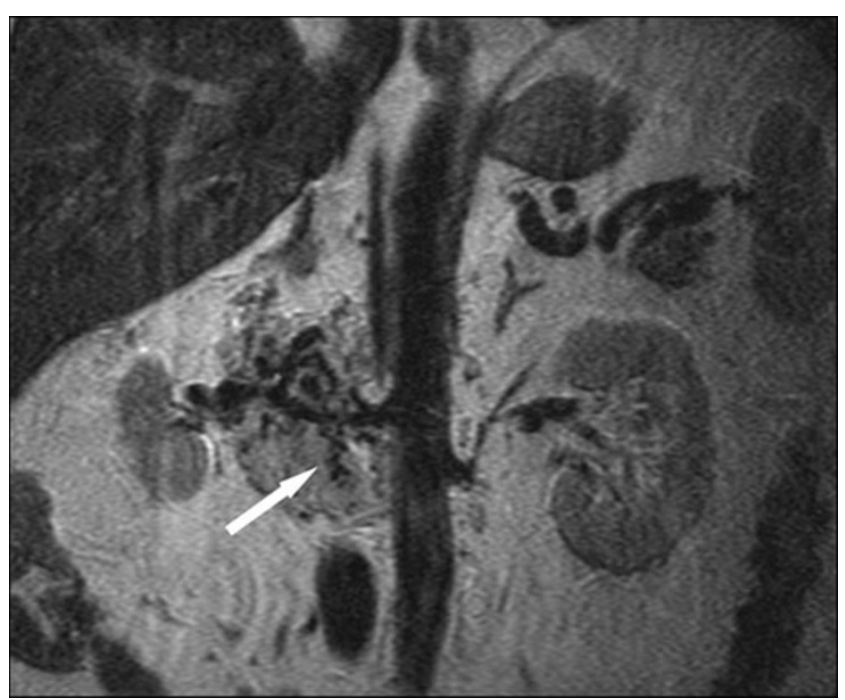

Fig. 3 A 60-year-old male patient with SDHB and a right-sided retroperitoneal paraganglioma. Coronal T2-weighted MR image demonstrating the intermediate to high $\mathrm{T} 2$-weighted signal intensity tumour enveloping the right renal artery. Note the signal voids (arrow) in the inferior portion of the tumour in keeping with the hypervascularity of the paraganglioma body tumours in patients with chronic hypoxia caused by disease or environmental factors such as living at high altitude $[5,6]$. It is, therefore, suspected that resistance to apoptosis due to mitochondrial dysfunction and pseudohypoxic drive are involved in tumourogenesis [7].

As early as the 1960s an awareness of the familial nature of some paragangliomas emerged, and within these families multiple tumours were commonly encountered [5]. In the 1990s Dutch researchers investigating paraganglioma families identified a region on chromosome 11 likely to be the area from within which the genetic mutations resulting in paraganglioma formation were to be found. This susceptibility locus on chromosome 11 was termed the "paraganglioma locus 1" or PGL1 [8]. As there was a known

\section{a}

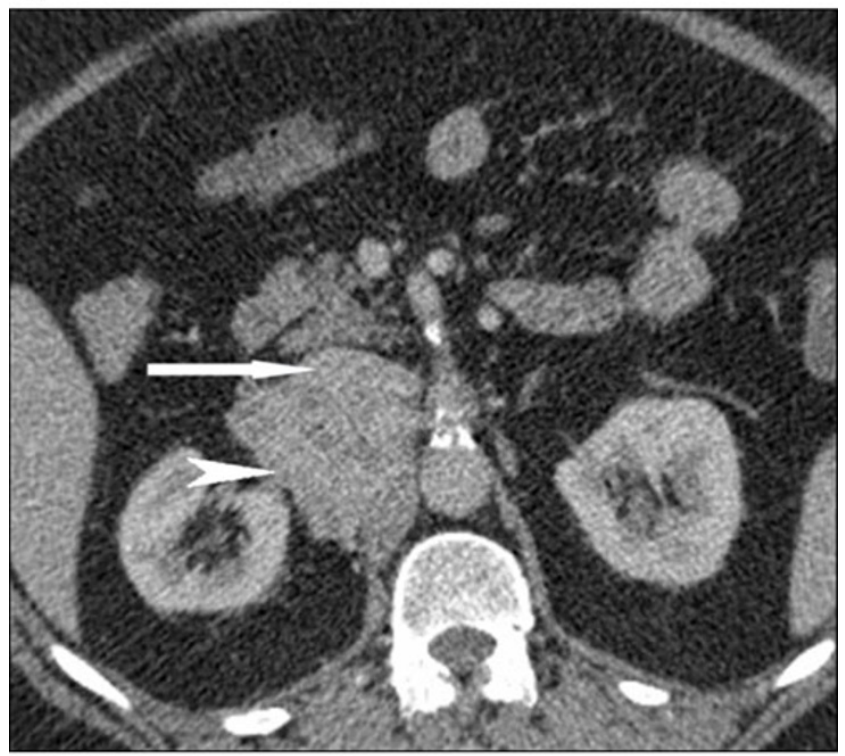

b

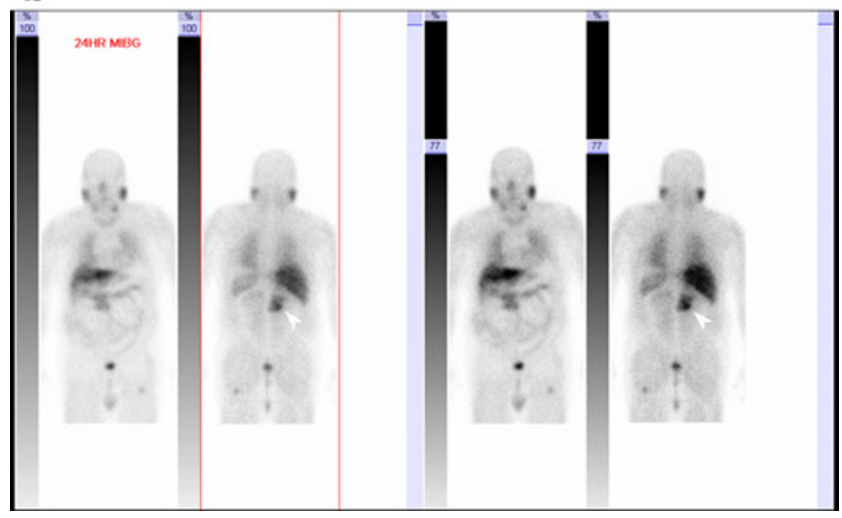

Fig. 4 A 60-year-old male patient with SDHB and a right-sided extraadrenal paraganglioma. a Contrast axial $\mathrm{CT}$ shows the right-sided para-aortic paraganglioma (arrowhead), which is displacing the IVC (arrow) anteriorly. b Methyl iodine benzyl guanidine (MIBG) scan of the same tumour showing avid uptake (arrowhead). MIBG, either labelled with ${ }^{123} \mathrm{I}$ or ${ }^{131} \mathrm{I}$, is a norepinephrine analogue. Sympathetic paragangliomas are usually secretory and therefore avidly take up MIBG as it follows the metabolic pathway of norepinephrine 
increased incidence of paragangliomas in people living at altitude [6], targeted investigation of the genes involved in aerobic metabolism in the PGL1 locus resulted in the discovery of the SDHD mutation [9].

Subsequent studies confirmed that SDHB and SDHC mutations also resulted in familial paragangliomas [10, 11]. SDHA mutation, however, has at present only been associated with metabolic neurodegenerative disorders [12].

The incidence of underlying SDH mutations in patients with apparently sporadic parasympathetic paragangliomas of the head and neck has been reported as high as $28 \%$ ( $7 \%$ due to SDHB, $4 \%$ SDHC and $17 \%$ SDHD) [12]. The incidence within sympathetic paragangliomas is approximately $10 \%$ (6\% due to SDHB and 4\% SDHD) [12].

\section{Inheritance patterns}

SDH mutations follow an autosomal dominant inheritance pattern. SDHD mutations are also subject to genomic imprinting of the maternal allele. Genomic imprinting of the maternal allele means the disease only manifests if the mutation is inherited from the father [13]. This may result in the disease skipping generations.

\section{Paraganglioma imaging characteristics summary}

\section{Ultrasound}

Ultrasound is commonly the first imaging modality used for investigation of neck masses. Paragangliomas of the neck appear as homogeneously hypoechoic masses. Multidirectional hypervascularity is the discerning feature on Doppler imaging (Fig. 1) [14].

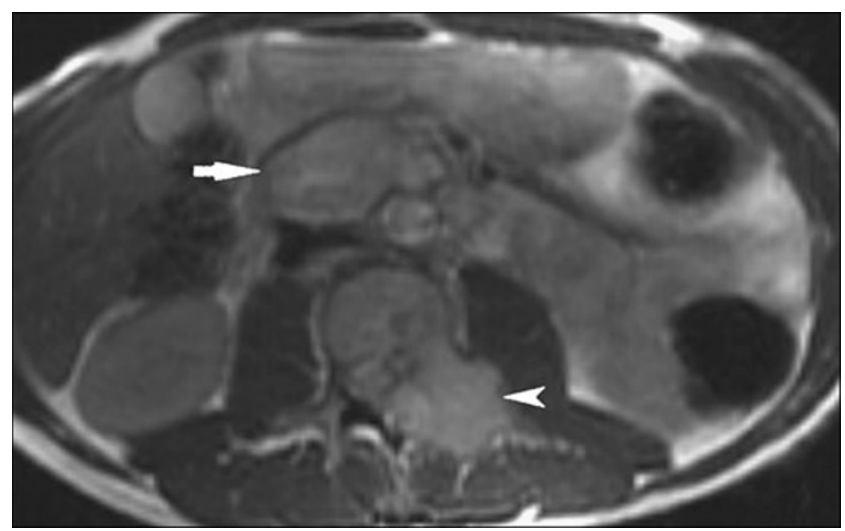

Fig. 5 A 42-year-old male patient with SDHB and a right-sided paraaortic paraganglioma and a spinal metastatic lesion. Axial T2weighted MRI image demonstrating the right para-aortic paraganglioma (arrow) with intermediate T2-weighted signal intensity. The spinal metastatic lesion (arrowhead) involves the pedicle of the vertebra and has the same signal intensity as the primary tumour
CT

Small paragangliomas appear as homogeneous masses, whereas larger paragangliomas can be heterogenous in appearance [15]. There is intense uniform enhancement following intravenous administration of contrast agent in keeping with the hypervascular nature of the tumour [16]. The location of the tumour aids further characterisation, e.g. carotid body tumours cause splaying of the internal and external carotid arteries, and jugulotympanic tumours result in bony erosion (Fig. 2). Punctate calcification or focal areas of high attenuation caused by acute haemorrhage may also be seen in some tumours [17].

\section{MRI}

Paragangliomas are usually of low to intermediate signal on T1-weighted images and intermediate to high signal on T2weighted images (Fig. 3). The hypervascularity results in multiple serpentine areas of signal void [18], which results in the characteristic "salt and pepper" appearance. The high signal areas, or "salt", are due to slow flow or haemorrhage, and the low signal areas, or "pepper", are due to the signal voids from high flow on T1- and T2-weighted sequences [19]. The high flow areas are better demonstrated on 3D time of flight sequences where they show high signal intensity [16]. As with CT, the tumours show avid homogeneous enhancement.

\section{SDHB presentation and tumour characteristics}

The precise incidence rate of SDH mutations is unknown. SDHB and SDHD mutations have similar prevalence and are more common compared to SDHC, which is rare [20].

SDHB mutation more commonly results in the formation of paragangliomas associated with the sympathetic system $[20,21]$. These are predominantly extra-adrenal paragangliomas of the thorax and abdomen (Fig. 4), and less commonly intra-adrenal paragangliomas or pheochromocytomas. There is an increased risk of malignant paragangliomas and metastatic disease with SDHB mutation (Fig. 5) $[21,22]$. Other than metastasis there are no specific features of malignancy; however, rapidity of growth and surrounding tissue invasion are suggestive. Extraparaganglial malignancies such as papillary thyroid carcinoma and renal cell carcinoma are also more prevalent in this group [20].

The age at paraganglioma diagnosis is reported at approximately 30 years [20-22].

SDHD presentation and tumour characteristics

SDHD mutations are associated with parasympathetic extra-adrenal paragangliomas [20,22]. This predominantly 

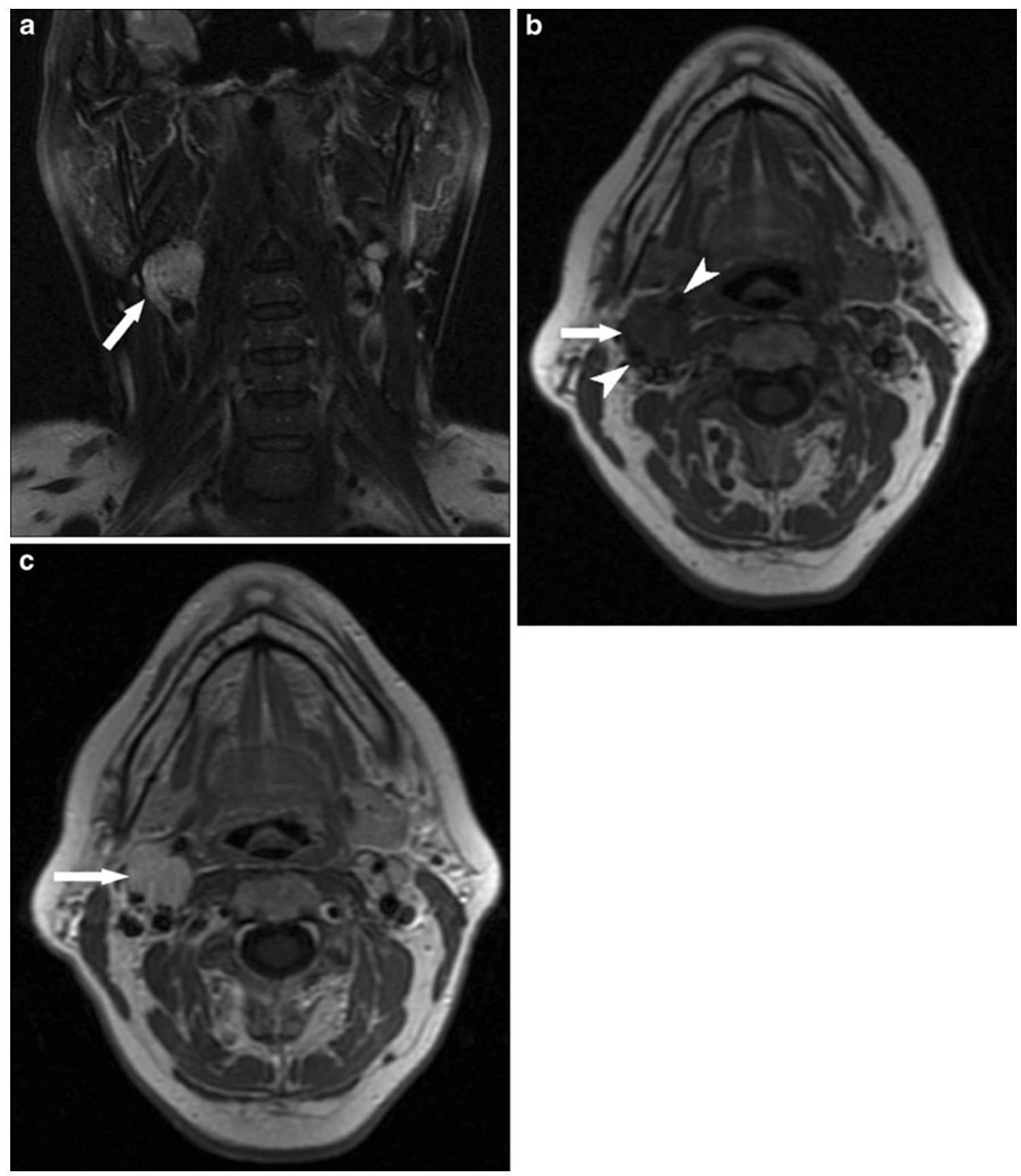

Fig. 6 A 56-year-old female patient with SDHD and a right-sided carotid paraganglioma of the neck; the same patient as in Fig. 1. a Coronal T2 fat saturated MR image demonstrating the right carotid paraganglioma (arrow) with high T2-weighted signal intensity. b Axial T1-weighted MR image showing the paraganglioma with low

results in head and neck tumours, which are usually benign (Fig. 6). There is an increased chance of multifocality (Figs. 7 and 8), but malignancy and metastatic disease are infrequent $[20,22]$. As with SDHB mutations, pheochromocytomas are less common.

The age at paraganglioma diagnosis is similar to SDHB at approximately 30 years [20,22].

T1-weighted single intensity (arrow). Note the splaying of the internal and external carotid arteries (arrowheads). c Gadolinium-enhanced axial T1-weighted MR image of the tumour showing avid uniform enhancement (arrow)

\section{SDHB versus SDHD}

Although SDHB and D mutations can be broadly divided into the categories above, it is important to remember that there can be overlap with tumour presentation. SDHB mutuations can result in head and neck paraganglioma formation, and SDHD mutations can develop thoracic and 

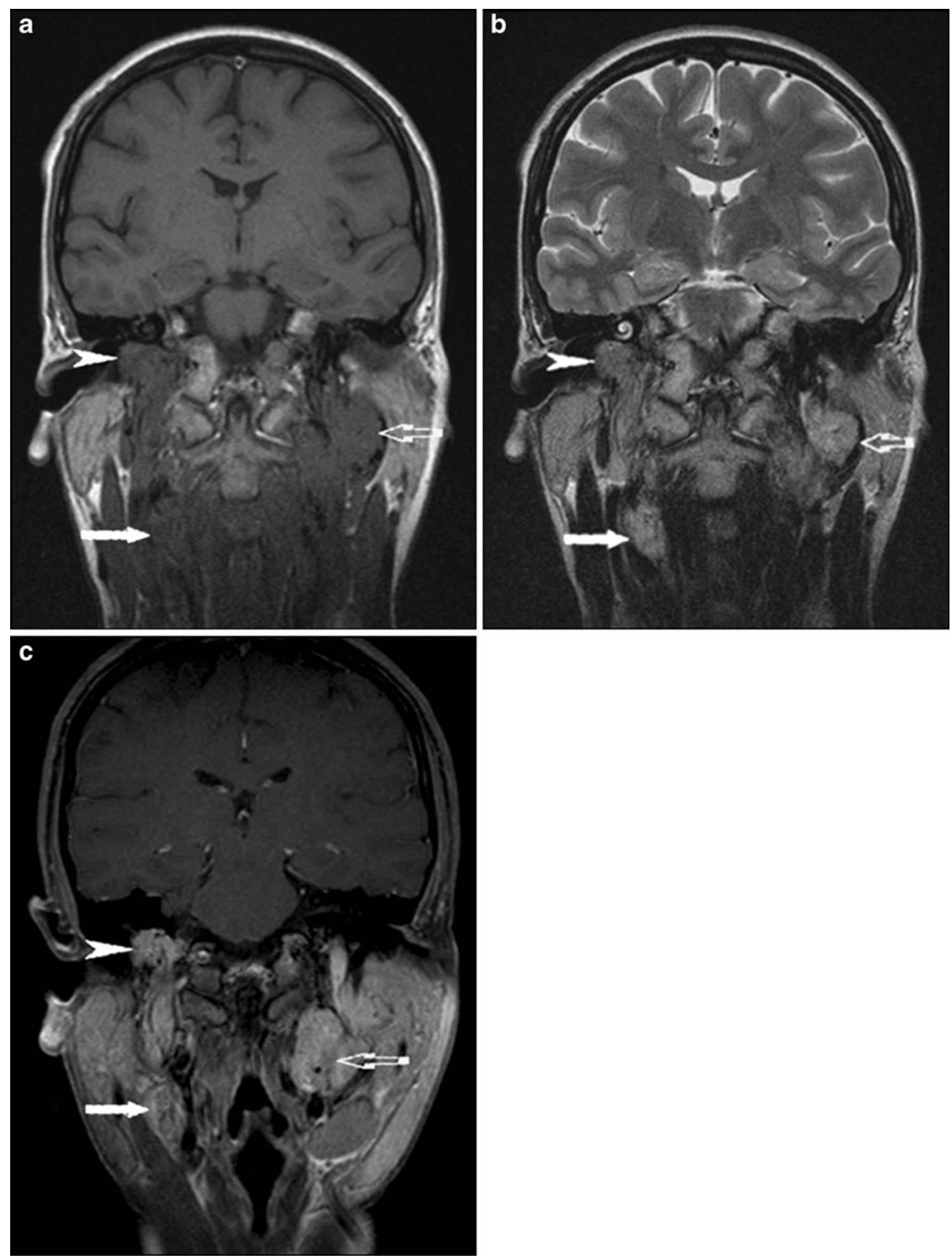

Fig. 7 A 35-year-old female patient with SDHD and multiple head and neck paragangliomas. a Coronal T1-weighted MR image demonstrating the right jugulotympanic (arrowhead), left carotid (open arrow) and right carotid paragangliomas (solid arrow). All three tumours are of low to intermediate T1-weighted signal intensity. b Coronal T2-weighted MR image demonstrating intermediate to high

signal of the right jugulotympanic (arrowhead), left carotid (open arrow) and right carotid paragangliomas (solid arrow). c Postgadolinium coronal T1-weighted MR image demonstrating avid homogeneous enhancement of the right jugulotympanic (arrowhead), left carotid (open arrow) and right carotid paragangliomas (solid arrow) 


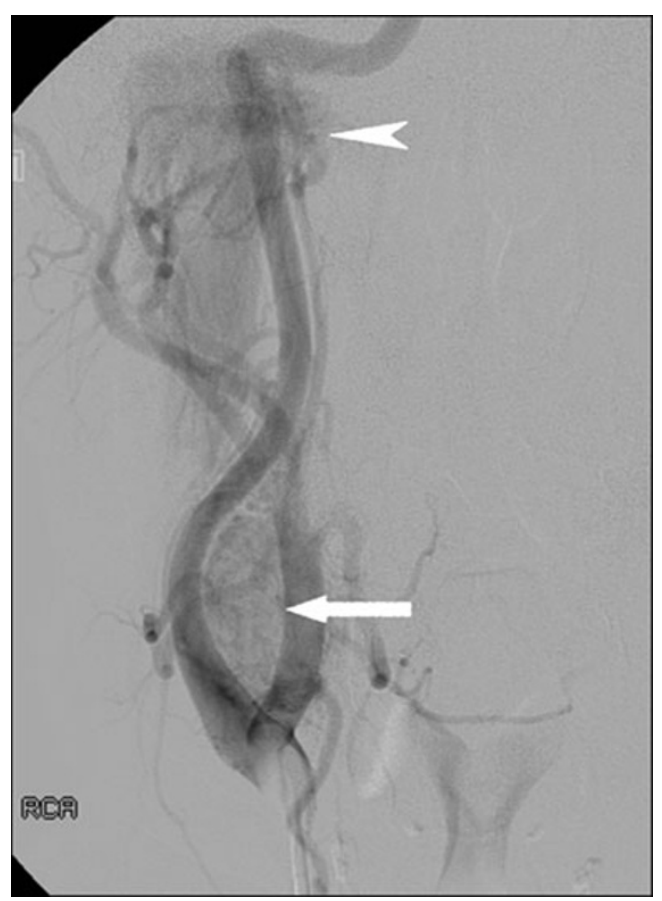

Fig. 8 Same patient as that in Fig. 7 with jugulotympanic and carotid paragangliomas. Angiography of the neck with catheterisation of the right common carotid artery. Note the increased vascularity at the site of the right jugulotympanic (arrowhead) and right carotid paragangliomas (arrow)

abdominal extra-adrenal paragangliomas [12, 23]. Both mutations can result in pheochromocytoma formation. The radiologist should be aware of this when reporting screening investigations for these patients.

\section{SDHA and SDHC}

SDHA mutations have been described in Leigh syndrome, a metabolic neurodegenerative disorder [24], and are not currently recognised to result in paraganglioma formation. SDHC mutations are rare and result in head and neck paraganglioma formation [12]. These tumours are usually benign and seldom multifocal [25]. Very rarely do SDHC mutations result in pheochromocytoma formation.

\section{SDHB and SDHD relevance}

The recent advances in genetics with regards to SDH mutations have implications for radiology. The radiological diagnosis of a paraganglioma or pheochromocytoma should result in a full genetic workup. Included in this is the imaging of susceptible family members for paragangliomas [7]. This can have a significant impact on the workload of a radiological department. SDH mutation-positive patients need ongoing screening as they are at high risk for developing paragangliomas, pheochromocytomas and further multifocal extraganglial tumours. In this context close collaboration with the local genetics department is essential. Following patient and family member interview and counselling, genetic mutation can be determined from a peripheral blood sample. The family history and type of tumour will determine which mutation is tested for initially.

Imaging protocols

A universal approach to screening is the subject of ongoing work. In a recent review article [12], Timmers et al. recommended MRI of the neck, chest, abdomen and pelvis every 1-2 years. This should be tailored according to individual needs and the specific mutation. In cases of SDHB mutation screening as early as 10 years of age is recommended. Further functional imaging may also be required along with annual history, examination and biochemical testing [26]. Local imaging guidelines should be developed in conjunction with the local genetics departments according to imaging availability and experience. Currently MRI of the head, neck, chest, abdomen and pelvis is the first line investigation in our practice. Subsequent follow-up may be performed with ultrasound or MRI. Post-treatment imaging in our institution is determined by the nature of the intervention on an individual patient basis.

\section{Conclusion}

SDHB and SDHD mutations result in paraganglioma formation. Whereas SDHB patients are prone to extraadrenal pheochromocytomas, malignant disease and extraparaganglial neoplasia, SDHD mutations have a greater propensity for multiple, benign head and neck paragangliomas. Diagnosis of a sporadic paraganglioma or pheochromocytoma should lead to a full genetic workup of the patient and family if SDH mutations are found. SDH mutation-positive family members should be placed on annual screening depending on the mutation. MRI of the neck, chest, abdomen and pelvis is the initial screening practice in our department. These mutations can therefore have a significant impact on radiologists and the resources of the radiology department.

\section{References}

1. Lack EE (1994) Paraganglioma. In: Sternberg SS (ed) Diagnostic Surgical Pathology, 2nd edn. Raven Press, New York, NY, pp 599-621

2. Rosai J (1996) Ackermans's surgical pathology, 8th edn. Mosby, New York, p 1015

3. van Gils AP, Falke TH, van Erkel AR et al (1991) MR imaging and MIBG scintigraphy of pheochromocytomas and extra adrenal functioning paragangliomas. Radiographics 11:37-57 
4. Pacak K, Eisenhofer G, Ahlman H et al (2007) Pheochromocytoma: recommendations for clinical practice from the First International Symposium (October 2005). Nat Clin Pract Endocrinol Metab 3:92-102

5. Martin TPC, Irving RM, Maher ER (2007) The genetics of paragangliomas: a review. Clin Otolaryngol 32:7-11

6. Pacheco-Ojeda LDL, Durango E, Rodriquez C et al (1988) Carotid body at high altitude. World J Surg 12:856-860

7. Favier J, Briere JJ, Strompf L et al (2005) Hereditary paraganglioma/Pheochromocytoma and inherited succinate dehydrogenase deficiency. Horm Res 63:171-179

8. Heutink P, van der Mey AG, Sandkujil LA et al (1992) A gene subject to genomic imprinting and responsible for hereditary paragangliomas maps to chromosome 11q23-qter. Hum Mol Genet 1:7-10

9. Baysal BE, Ferrell RE, Willet-Brozick JE et al (2000) Mutations in SDHD, a mitochondrial complex II gene, in hereditary paraganglioma. Science 287:848-851

10. Astuti D, Latif F, Dallol A et al (2001) Gene mutations in the succinate dehydrogenase subunit SDHB cause susceptibility to familial pheochromocytoma and to familial paraganglioma. Am J Hum Genet 69:49-54

11. Taschner PE, Jansen JC, Baysal BE, et al. (2001) Nearly all hereditary paragangliomas in the Netherlands are caused by two founder mutations in the SDHD gene. Genes Chromosomes Cancer 274-281

12. Timmers HJ, Gimenez-Roqueplo AP, Mannelli M et al (2009) Clinical aspects of SDHx-related pheochromocytoma and paraganglioma. Endocrine -Related. Cancer 16:391-400

13. Van der Mey AG, Maaswinkel-Mooy PD, Cornelisse CJ et al (1989) Genomic imprinting in hereditary glomus tumours: Evidence for new genetic theory. Lancet 2:1291-1294

14. Jansen JC, Baatenburg de Jong RJ, Schipper J et al (1997) Colour Doppler imaging of paragangliomas of the neck. J Clin Ultrasound 25(9):481-485
15. Sahdev A, Sohaib A, Monson JP et al (2005) CT and MR imaging of unusual locations of extra-adrenal paragangliomas (pheochromocytomas). Eur Radiol 15:85-92

16. Van den Berg R (2005) Imaging and management of head and neck paragangliomas. Eur Radiol 15:1310-1318

17. Hayes WS, Davidson AJ, Grimley PM et al (1990) Extraadrenal retroperitoneal paraganglioma: clinical, pathologic and $\mathrm{CT}$ findings. AJR Am J Roentgenol 155:1247-1250

18. Lee KY, Oh YW, Noh HJ et al (2006) Extraadrenal paragangliomas of the body: imaging features. AJR Am J Roentgenol 187:492-504

19. Olsen WL, Dillon WP, Kelly WM et al (1987) MR imaging of paragangliomas. AJR Am J Roentgenol 148:201-204

20. Neuman HP, Pawlu C, Peczkowska M et al (2004) Distinct clinical features of paraganglioma syndromes associated with SDHB and SDHD gene mutations. JAMA 292(8):943-952

21. Timmers HJ, Kouzupa A, Eisenhofer G et al (2007) Clinical presentations, biochemical phenotypes, and genotype-phenotype correlations in patients with succinate dehydrogenase subunit Bassociated phaechromomcytomas and paragangliomas. J Clin Endocrinol Metab 92(3):779-786

22. Benn DE, Gimenez-Roqueplo AP, Reilly JR et al (2006) Clinical presentation and prevalence of pheochromocytoma/paraganglioma syndromes. J Clin Endocrinol Metab 91(3):827-836

23. Adler JT, Meyer-Rochow GY, Chen H et al (2008) Pheochromocytoma: Current Approaches and Future Directions. Oncologist 13:779-793

24. Horváth R, Abicht A, Holinski-Feder E et al (2006) Leigh syndrome caused by mutations in the flavoprotein ( $\mathrm{Fp}$ ) subunit of succinate dehydrogenase (SDHA). J Neurol Neurosurg Psychiatry 77(1):74-76

25. Schiavi F, Boedeker CC, Bausch B et al (2005) Predictors and prevalence of paraganglioma syndrome associated with mutations of the SDHC gene. JAMA 294(16):2057-2063

26. Young WF, Abboud AL (2006) Editorial: Paraganglioma-All in the family. J Clin Endocrinol Metab 91(3):790-792 